\title{
Lactate and Bilirubin Index: A New Indicator to Predict Critically Ill Cirrhotic Patients' Prognosis
}

\author{
Xiao-Fu Chen $(\mathbb{D}$, Yuan Zhao $\mathbb{D}$, Wei-Zhen Chen $\mathbb{D}$, Xin-Tian Shao $\mathbb{D}$, \\ and Zhi-Ming Huang \\ Department of Gastroenterology and Hepatology, The First Affiliated Hospital of Wenzhou Medical University, \\ Wenzhou 325000, China \\ Correspondence should be addressed to Zhi-Ming Huang; wyhuangzhiming@126.com
}

Received 24 December 2020; Revised 24 December 2020; Accepted 3 February 2021; Published 12 February 2021

Academic Editor: Alessandro Granito

Copyright ( $\odot 2021$ Xiao-Fu Chen et al. This is an open access article distributed under the Creative Commons Attribution License, which permits unrestricted use, distribution, and reproduction in any medium, provided the original work is properly cited.

Objectives. We aimed to perform external validation of the prognostic value of the lactate and bilirubin (LB) index, a new indicator, and compare the ability of the LB index and other scoring systems to predict both short- and long-term mortality in critically ill cirrhotic patients. Materials and Methods. A number of 479 cirrhotic patients admitted into ICU were included in our research. We measured prognostic scores in the first 24 hours including LB index, Child-Pugh, SOFA, CLIF-SOFA, and MELD scores. The LB index was calculated as follows: $\ln [1000 \times$ lactate $(\mathrm{mmol} / \mathrm{L}) \times$ bilirubin $(\mu \mathrm{mol} / \mathrm{L})] / 2$. The primary outcomes were 28 -day and 3-year all-cause mortality. Multivariate logistic regression analyses were used to investigate the independent association between the LB index and the mortality in critically ill cirrhotic patients. The area under the receiver operating characteristic curve was used to assess the prediction accuracy of short- and long-term mortality of the clinical score. Calibration of the score was evaluated by Hosmer-Lemeshow goodness-of-fit test for significance. Results. Multivariate logistic regression analysis identified that the LB index (odds ratio: 5.487, 95\% confidence interval: 3.542-8.501, $P<0.001$ ) was the strongest predictor for 28-day mortality. The LB index gave the highest area under the curve $(0.791,95 \%$ confidence interval: $0.747-0.836)$ in predicting 28 -day mortality. For predicting 3year mortality, the model for end-stage liver disease (MELD) score showed better discrimination ability with an area under the curve of 0.726 (95\% confidence interval: $0.680-0.771$ ). The risk of mortality significantly increased when the clinical scores were $\geq$ the optimal cutoff values. Conclusions. The LB index, a simple prognostic indicator, performs well in predicting critically ill cirrhotic patients' short-term prognosis, while, for long-term prognosis, the MELD score is more appropriate.

\section{Introduction}

Cirrhotic patients often require entering an intensive care unit (ICU) due to life-threatening complications [1-3]. Decompensated cirrhotic patients are often accompanied by progressive organ failure [4, 5]. According to statistics, hospital mortality of cirrhotic patients in ICU ranges from 34 to $86 \%$ [4]. Various clinical models have been developed to evaluate the severity of cirrhosis accurately so as to determine the optimum therapeutic regimen, including Child-Pugh system [6], sequential organ failure assessment(SOFA) [7], chronic liver failure-sequential organ failure assessment (CLIF-SOFA) [8], and model for endstage liver disease (MELD) score [9]. They were externally validated in cirrhotic patients entering ICU [10-12].
Lactate production increases and utilization decreases on account of tissue hypoxia and impaired mitochondrial oxidation [13]. Yet, up to seventy percent of whole-body lactate is cleared in the liver $[14,15]$. Increased lactate levels are not only associated with acute hepatic impairment, but also independently associated with critically ill cirrhotic patients' mortality [16-20]. Besides, total bilirubin is an important index of liver function. Therefore, we proposed the lactate and bilirubin (LB) index, a new indicator to predict critically ill cirrhotic patients' prognosis, which was convenient and practical compared to other scoring systems. In addition, we discovered that little was known about the value of the LB index and the scoring systems mentioned above in predicting critically ill cirrhotic patients' short- and long-term prognosis. Thus, our research's objective was to perform 
external validation of the prognostic value of the LB index and compare the ability of the LB index and the scoring systems mentioned above to predict both short- and longterm mortality in critically ill cirrhotic patients.

\section{Materials and Methods}

2.1. Data Source. The Medical Information Mart for Intensive Care III is an open-access database, including information about patients who entered ICU between 2001 and 2012 [21]. After finalization of the National Institute of Health' $s$ training course named "Protecting Human Research Participants," we granted access to the database (certificate number: 36072928). The institutional review boards of the Massachusetts Institute of Technology had approved the establishment. Ethics committee approval was not necessary for this manuscript. The research was a retrospective observational research, all information relating to patients in the database anonymous; therefore, informed consent was not necessary.

2.2. Study Population. Patients who were at least 18 years old at their first ICU admission and stayed more than $24 \mathrm{~h}$ were selected. The diagnosis of liver cirrhosis was based on the following features: (a) characteristic clinical evidence such as jaundice, (b) abnormal laboratory findings such as thrombocytopenia, (c) imaging findings such as signs of portal hypertension, and (d) histopathology [22, 23]. Exclusion criteria were as follows: (a) acquired immune deficiency syndrome, (b) carcinoma, (c) liver transplantation, and (d) total bilirubin or lactate data lost at ICU admission. After applying exclusion criteria, the study sample group included 479 patients with cirrhosis.

2.3. Data Collection. Clinical parameters we collected from the database included demographic data, vital signs, and laboratory parameters. Laboratory parameters included white blood cell count, hemoglobin, platelet count, prothrombin time, glucose, blood urea nitrogen, creatinine, albumin, total bilirubin, and arterial blood lactate. The average values of each parameter within the first $24 \mathrm{~h}$ after being admitted to ICU were used to calculate the scores of clinical models. Child-Pugh, SOFA, CLIF-SOFA, and MELD scores were calculated using the formulae published [6-9]. The LB index was calculated as follows: In $[1000 \times$ lactate $(\mathrm{mmol} / \mathrm{L}) \times$ bilirubin $(\mu \mathrm{mol} / \mathrm{L})] / 2$. The start date for follow-up was the date of admission. All participants were followed up for at least 3 years. The outcomes of our research were 28-day, 90-day, 1-year, and 3-year all-cause mortality. The primary endpoints were 28-day and 3-year all-cause mortality.

2.4. Statistical Analysis. All statistical procedures were performed with STATA (version 14.0; StataCorp, State of Texas, USA). Quantitative variables were compared by Mann-Whitney $U$ test, presented as median [interquartile range (IQR)]. Categorical variables were expressed absolute numbers (frequencies) and compared by Chi-square test or Fisher's exact test. Multivariate logistic regression analyses were used to investigate the independent association between various clinical parameters and mortality in critically ill cirrhotic patients, while controlling for potential confounding. Odds ratio (OR) was reported with 95\% confidence interval (CI). Receiver operating characteristic curves were generated to evaluate the accuracy of clinical models in predicting mortality. Calibration of the score was evaluated by Hosmer-Lemeshow goodness of fit test for significance $(P>0.05)$. The comparison between the area under the curve (AUC) was performed using DeLong test [24]. The sensitivity and specificity at an optimal cutoff value were compared among different clinical models. All the participants were divided into two groups (relatively low risk and high risk) by the optimal cutoff value of clinical scores according to the curve. All the tests were two-sided. A $P$ value less than 0.05 was considered statistically significant.

\section{Results}

3.1. Baseline Characteristics of the Study Population. A number of 479 participants were included in our research. The baseline characteristics of the participants in both survivor cohort and nonsurvivor cohort are listed in Tables 1 and 2 in detail. All the patients' median age was 55.6 years; 315 were male and 164 were female. The majority of participants were white. The predominant cause of cirrhosis was alcohol. A total of 60 patients had two causes of cirrhosis and three patients had three. Infection and sepsis were the most common causes of ICU hospitalization. The survivor and nonsurvivor cohorts were similar in age, sex, weight, comorbidity, length of ICU stay, hemoglobin, glucose, and albumin $(P \geq 0.05)$. Nonsurvivors scored higher than survivors in these clinical scores including LB index, MELD, Child-Pugh, SOFA, and CLIF-SOFA scores (all $P<0.001)$.

\subsection{Results of Multivariate Logistic Regression Analysis.} The results of parameters significant in the multivariate logistic regression are shown in Table 3. As the results show, the LB index was the strongest predictor for 28-day mortality. Furthermore, age, length of ICU stay, mechanical ventilation duration, BUN, PTT, and respiratory rate were also identified as independent risk factors for 28-day mortality in cirrhotic patients entering ICU.

\subsection{Comparison of Different Scoring Systems in Predicting 28-} Day and 3-Year Mortality in Critically Ill Cirrhotic Patients. Figure 1 shows that the LB index performed the best in predicting 28-day mortality. Figure 2 shows that the performance of LB index, Child-Pugh, SOFA, and CLIF-SOFA scores was worse while the MELD score still performed well in predicting 3-year mortality. More details about the performance of different scoring systems are presented in Tables 4 and 5. The calibration curve of the LB index for 28day mortality is shown in Figure $3(P=0.728)$. 
TABLE 1: Characteristics of the study population, stratified by survival.

\begin{tabular}{|c|c|c|c|c|}
\hline Parameter & All patients $(n=479)$ & Survivors $(n=321)$ & Nonsurvivors $(n=158)$ & $P$ value \\
\hline Age (years) & $55.6(48.9-64.8)$ & $55.8(48.3-65.2)$ & $55.2(49.9-64.3)$ & 0.710 \\
\hline Sex: male & $315(65.8)$ & $213(66.4)$ & $102(64.6)$ & 0.697 \\
\hline Weight (kg) & $85.3(70.9-99.0)$ & $85.3(70.5-97.5)$ & $85.0(71.2-100.0)$ & 0.660 \\
\hline \multicolumn{5}{|l|}{ Ethnicity } \\
\hline White & $350(73.1)$ & $241(75.1)$ & $109(69.0)$ & 0.041 \\
\hline Black & $31(6.5)$ & $24(7.5)$ & $7(4.4)$ & \\
\hline Others & $98(20.5)$ & $56(17.4)$ & $42(26.6)$ & \\
\hline \multicolumn{5}{|l|}{ Causes of cirrhosis } \\
\hline Alcoholic & $264(55.1)$ & $167(52.0)$ & $97(61.4)$ & 0.053 \\
\hline Hepatitis B & $12(2.5)$ & $5(1.6)$ & $7(4.4)$ & 0.059 \\
\hline Hepatitis C & $119(24.8)$ & $77(24.0)$ & $42(26.6)$ & 0.537 \\
\hline Biliary & $9(1.9)$ & $0(0.0)$ & $9(5.7)$ & 0.034 \\
\hline Autoimmune & $5(1.0)$ & $3(0.9)$ & $2(1.3)$ & 0.667 \\
\hline Others & $136(28.4)$ & $103(32.1)$ & $33(20.9)$ & 0.011 \\
\hline \multicolumn{5}{|l|}{ Primary cause of ICU admission } \\
\hline Infection/sepsis & $132(27.6)$ & $93(29.0)$ & $39(24.7)$ & 0.323 \\
\hline Bleeding & $107(22.3)$ & $79(24.6)$ & $28(17.7)$ & 0.089 \\
\hline Respiratory & $17(3.5)$ & $12(3.7)$ & $5(3.2)$ & 0.750 \\
\hline Cardiovascular & $56(11.7)$ & $42(13.1)$ & $14(8.9)$ & 0.176 \\
\hline Renal failure & $25(5.2)$ & $12(3.7)$ & $13(8.2)$ & 0.038 \\
\hline Neurological failure & $49(10.2)$ & $31(9.7)$ & $18(11.4)$ & 0.556 \\
\hline Others & $93(19.4)$ & $52(16.2)$ & $41(25.9)$ & 0.011 \\
\hline \multicolumn{5}{|l|}{ Comorbidity } \\
\hline Hypertension & $122(25.5)$ & $80(24.9)$ & $42(26.6)$ & 0.695 \\
\hline Diabetes & $124(25.9)$ & $85(26.5)$ & $39(24.7)$ & 0.673 \\
\hline \multicolumn{5}{|l|}{ Vital signs } \\
\hline Temperature $\left({ }^{\circ} \mathrm{C}\right)$ & $36.7(36.3-37.2)$ & $36.8(36.4-37.3)$ & $36.4(36.0-37.0)$ & $<0.001$ \\
\hline Heart rate & $90.4(78.7-103.2)$ & $88.6(76.8-101.1)$ & $94.2(81.1-105.4)$ & 0.021 \\
\hline MAP (mmHg) & $73.1(67.9-80.7)$ & $74.8(69.4-82.5)$ & $70.0(64.4-75.6)$ & $<0.001$ \\
\hline Respiratory rate & $18.9(16.1-22.1)$ & $18.3(15.9-21.0)$ & $20.2(17.3-23.7)$ & $<0.001$ \\
\hline $\mathrm{SpO} 2 / \mathrm{FiO} 2$ & $185.2(173.1-457.0)$ & $204.6(175.2-458.1)$ & $175.8(169.4-455.8)$ & $<0.001$ \\
\hline 24 -h urine output $(\mathrm{mL})$ & $1151(572-1915)$ & $1360(787-2028)$ & $653(222-1280)$ & $<0.001$ \\
\hline Mechanical ventilation duration (hours) & $36.0(0.0-120.5)$ & $19.0(0.0-112.5)$ & $60.1(12.0-146.5)$ & $<0.001$ \\
\hline Length of ICU stay (days) & $4.0(2.4-8.5)$ & $3.8(2.3-8.0)$ & $4.7(2.8-9.0)$ & 0.147 \\
\hline
\end{tabular}

Values are expressed as $n$ (\%) or median (IQR). ICU: intensive care unit; MAP: mean arterial pressure; SpO2: peripheral oxygen saturation; FiO2: fraction of inspired oxygen.

3.4. Groups and Outcomes. The 28-day, 90-day, 1-year, and 3 -year death rates of all the participants were $33.0 \%$ (158/ 479 ), $41.8 \%$ (200/479), $48.6 \%$ (233/479), and 57.6\% (276/ $479)$, respectively. On the basis of the LB index classification (low risk: $<6.18$ and high risk: $\geq 6.18$ ), the 28 -day, 90 day, 1 -year, and 3 -year death rates for the patients with low risk were $16.0 \%$ (48/300), 24.7\% (74/300), 33.0\% (99/300), and $44.7 \%(134 / 300)$, respectively, and for the patients with high risk, 61.5\% (110/179), 70.4\% (126/179), 74.9\% (134/ 179 ), and $79.3 \%(142 / 179)$, respectively (all $P<0.001$ ), while, on the basis of the MELD classification (low risk: $<18$ and high risk: $\geq 18$ ), the 28-day, 90-day, 1-year, and 3-year death rates were $16.9 \%$ (45/266), $23.7 \%$ (63/266), $32.0 \%$ (85/266), and $42.9 \%$ (114/266), respectively, for the patients with low risk, and $53.1 \%$ (113/213), $64.3 \%$ (137/213), $69.5 \%$ $(148 / 213)$, and $76.1 \%(162 / 213)$, respectively, for the patients with high risk (all $P<0.001$ ). The risk of mortality significantly increased when the clinical scores were $\geq$ the cutoff values.

\section{Discussion}

In the research, we performed for the first time external validation of the prognostic value of blood LB index and compared for the first time the ability of the LB index, Child-Pugh, MELD, SOFA, and CLIF-SOFA scores to predict both short- and long-term mortality in critically ill cirrhotic patients.

As this study shows, mortality in critically ill patients with cirrhosis was high, which was comparable to the previous reports $[4,5,25,26]$. We found that cirrhotic patients in ICU had poor outcomes despite aggressive medical interventions. Therefore, in order to determine the best treatment timely and improve long-term quality of life, it is quite necessary to assess the severity of disease and longterm prognosis in critically ill cirrhotic patients.

The Child-Pugh score was originally developed to determine the risk of surgery for portal decompression in cirrhotic patients, which was also used to assess prognosis of 
TABLE 2: Laboratory parameters and clinical scores of the study population, stratified by survival.

\begin{tabular}{|c|c|c|c|c|}
\hline Parameter & All patients $(n=479)$ & Survivors $(n=321)$ & Nonsurvivors $(n=158)$ & $P$ value \\
\hline \multicolumn{5}{|c|}{ Laboratory parameters } \\
\hline $\mathrm{Hb}(\mathrm{mg} / \mathrm{dL})$ & $9.9(9.0-11.1)$ & $10.0(9.1-11.2)$ & $9.8(8.8-11.0)$ & 0.104 \\
\hline WBC $\left(10^{9} / \mathrm{L}\right)$ & $10.5(7.4-15.7)$ & $10.2(7.3-14.4)$ & $11.6(7.7-19.1)$ & 0.007 \\
\hline Platelet $\left(10^{9} / \mathrm{L}\right)$ & $101.2(70.3-152.0)$ & $110.2(74.3-162.0)$ & $88.5(62.3-129.0)$ & $<0.001$ \\
\hline INR & $1.7(1.5-2.1)$ & $1.6(1.4-1.9)$ & $2.0(1.7-2.7)$ & $<0.001$ \\
\hline PT (seconds) & $18.0(15.6-21.4)$ & $16.8(15.3-19.2)$ & $20.6(17.5-24.6)$ & $<0.001$ \\
\hline PTT (seconds) & $39.5(33.6-48.7)$ & $37.4(32.4-43.5)$ & $45.3(38.4-62.7)$ & $<0.001$ \\
\hline Glucose (mg/dL) & $125.6(103.0-157.8)$ & $125.6(103-156.5)$ & $124.4(101.2-158.0)$ & 0.803 \\
\hline Sodium $(\mathrm{mEq} / \mathrm{L})$ & $138.0(134.0-141.0)$ & $138.3(135.6-141.2)$ & $136.0(132.0-141.0)$ & $<0.001$ \\
\hline Potassium(mEq/L) & $4.1(3.7-4.5)$ & $4.1(3.7-4.4)$ & $4.3(3.8-4.8)$ & 0.001 \\
\hline BUN (mg/dL) & $29.5(17.0-50.0)$ & $25.3(15.5-42.3)$ & $41.7(26.5-63.3)$ & $<0.001$ \\
\hline Creatinine $(\mathrm{mg} / \mathrm{dL})$ & $1.3(0.8-2.5)$ & $1.1(0.7-1.9)$ & $1.9(1.2-3.3)$ & $<0.001$ \\
\hline Albumin $(\mathrm{g} / \mathrm{dL})$ & $2.8(2.6-3.0)$ & $2.8(2.6-3.0)$ & $2.8(2.5-3.1)$ & 0.702 \\
\hline Bilirubin (mg/dL) & $3.3(1.6-7.6)$ & $2.7(1.3-4.6)$ & $7.3(3.2-16.1)$ & $<0.001$ \\
\hline Lactate $(\mathrm{mmol} / \mathrm{L})$ & $2.2(1.6-3.7)$ & $2.0(1.5-3.0)$ & $2.9(2.0-6.0)$ & $<0.001$ \\
\hline \multicolumn{5}{|l|}{ Clinical scores } \\
\hline Child-Pugh & $10(9-11)$ & $10(9-11)$ & $11(10-11)$ & $<0.001$ \\
\hline SOFA & $9(6-11)$ & $8(6-10)$ & $11(9-14)$ & $<0.001$ \\
\hline CLIF-SOFA & $9(7-12)$ & $9(6-10)$ & $12(10-15)$ & $<0.001$ \\
\hline MELD & $16(10-25)$ & $13(8-20)$ & $24(16-33)$ & $<0.001$ \\
\hline LB index & $5.9(5.4-6.5)$ & $5.7(5.3-6.1)$ & $6.6(5.9-7.0)$ & $<0.001$ \\
\hline
\end{tabular}

Values are expressed as $n$ (\%) or median (IQR). Hb: hemoglobin; WBC: white blood cell; INR: international normalized ratio; PT: prothrombin time; PTT: partial thromboplastin time; BUN: blood urea nitrogen; SOFA: sequential organ failure assessment; CLIF-SOFA: chronic liver failure-sequential organ failure assessment; MELD: model for end-stage liver disease; LB: lactate and bilirubin.

TABLE 3: Multivariate regression results of 28-day mortality for critically ill patients with cirrhosis.

\begin{tabular}{lcccc}
\hline \multirow{2}{*}{ Parameter } & \multirow{4}{c}{$95 \%$ CI } & \multirow{2}{*}{ OR value } \\
& & Lower & Upper & \\
\hline Age (years) & 1.034 & 1.012 & 1.057 & 0.002 \\
Length of ICU stay (days) & 0.860 & 0.785 & 0.942 & 0.001 \\
Mechanical ventilation duration & 1.006 & 1.002 & 1.010 & 0.005 \\
(hours) & & & & \\
BUN (mg/dL) & 1.015 & 1.005 & 1.025 & 0.002 \\
PTT (seconds) & 1.032 & 1.017 & 1.048 & $<0.001$ \\
Respiratory rate & 1.069 & 1.015 & 1.127 & 0.012 \\
LB index & 5.487 & 3.542 & 8.501 & $<0.001$ \\
\hline
\end{tabular}

ORs and $P$ values were estimated using multivariate logistic regression. Age, sex, length of ICU stay, and variables were statistically significant in the tests and were included in the multivariate analysis. OR: odds ratio; CI: confidence interval; ICU: intensive care unit; BUN: blood urea nitrogen; PTT: partial thromboplastin time; LB: lactate and bilirubin.

liver cirrhosis [6]. SOFA was an effective predictor of mortality in patients admitted to ICU [27]. CLIF-SOFA performed well in predicting 6-month mortality in critically ill cirrhotic patients [11]. The MELD score was used to predict survival in patients with end-stage liver disease [9].

Lactate can be measured in critically ill patients to evaluate the severity of disease [28-30]. Lactate metabolism in cirrhotic patients was significantly different from that in patients without liver damage, leading to a net increase in lactate levels $[14,31]$. Increased lactate levels were associated with poor outcome in cirrhotic patients admitted to ICU $[17,32,33]$. The total bilirubin was a significant indicator of liver function. Total bilirubin level was included as an indispensable factor in MELD, Child-Pugh, SOFA, and CLIF-SOFA scores. Thus, we proposed the LB index, a new

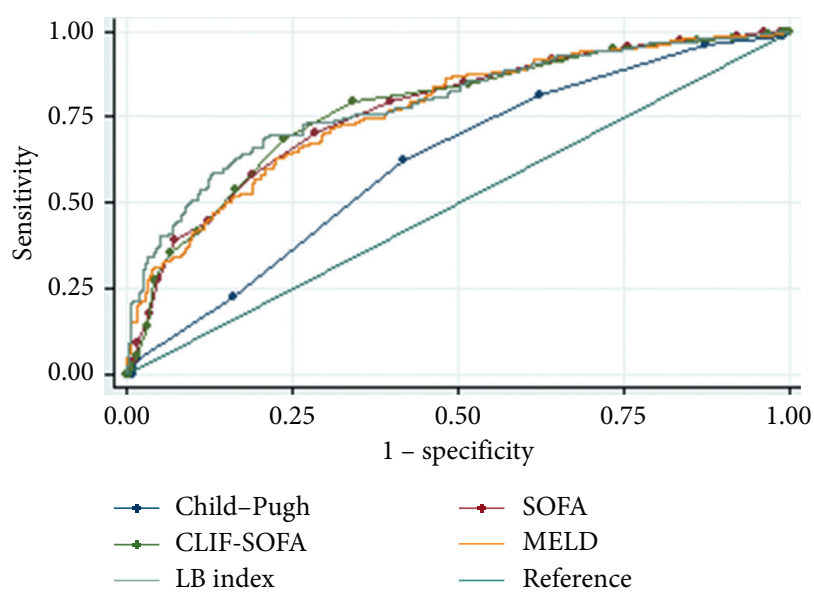

FIGURE 1: Area under the receiver operating characteristic curve of the scoring systems in predicting 28-day mortality in critically ill patients with cirrhosis. SOFA, sequential organ failure assessment; CLIF-SOFA, chronic liver failure-sequential organ failure assessment; MELD, model for end-stage liver disease; LB: lactate and bilirubin.

indicator to predict critically ill cirrhotic patients' prognosis and externally validated it's ability. As our research finally demonstrated, the LB index was an independent predictive factor of poor prognosis of cirrhotic patients.

The laboratory-based LB index has its unique advantages. Through noninvasive blood testing, these values can be readily obtained and objectively evaluated. Besides, compared to other scoring systems built for cirrhotic patients, the LB index can be easily and rapidly calculated at patients' bedside using a simple and convenient formula. Moreover, despite its simplicity, this clinical indicator still 


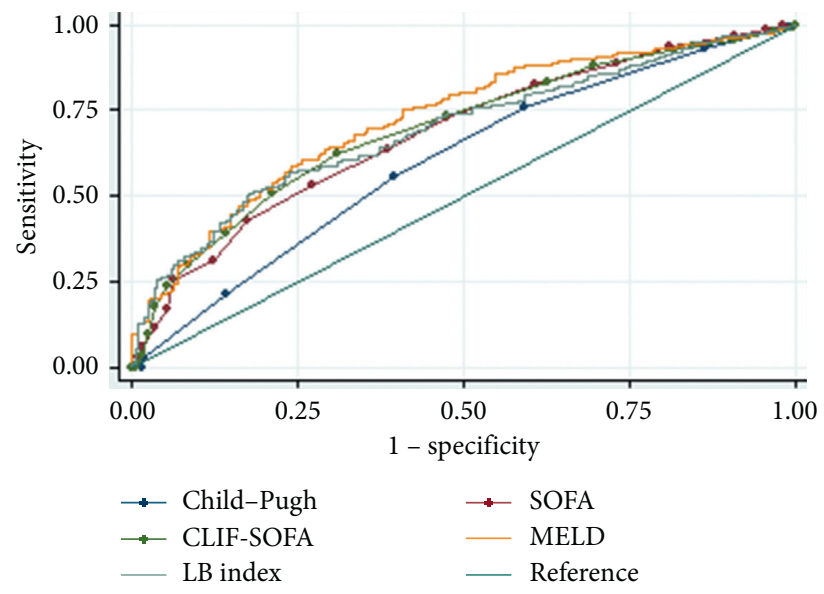

FIGURE 2: Area under the receiver operating characteristic curve of the scoring systems in predicting 3-year mortality in critically ill patients with cirrhosis. SOFA, sequential organ failure assessment; CLIF-SOFA, chronic liver failure-sequential organ failure assessment; MELD, model for end-stage liver disease; LB: lactate and bilirubin.

TABle 4: Diagnostic accuracy of different scoring systems in predicting 28-day mortality at the optimal cutoff point.

\begin{tabular}{|c|c|c|c|c|c|c|c|c|c|}
\hline Prognostic models & AUROC (95\% CI) & $P$ value & Cutoff point & Sensitivity & Specificity & PPV & NPV & PLR & NLR \\
\hline LB index & $0.791(0.747-0.836)$ & - & 6.18 & 0.70 & 0.79 & 0.61 & 0.84 & 3.24 & 0.39 \\
\hline Child-Pugh & $0.624(0.574-0.674)$ & $<0.001$ & 11 & 0.63 & 0.58 & 0.42 & 0.76 & 1.50 & 0.64 \\
\hline SOFA & $0.771(0.727-0.816)$ & 0.372 & 10 & 0.70 & 0.72 & 0.55 & 0.83 & 2.48 & 0.42 \\
\hline CLIF-SOFA & $0.775(0.731-0.820)$ & 0.455 & 10 & 0.80 & 0.66 & 0.53 & 0.87 & 2.33 & 0.31 \\
\hline MELD & $0.768(0.723-0.813)$ & 0.271 & 18 & 0.72 & 0.69 & 0.53 & 0.83 & 2.32 & 0.40 \\
\hline
\end{tabular}

DeLong test was used to compare the AUC between LB index and other clinical models. AUROC: area under the receiver operating characteristic curve; CI: confidence interval; PPV: positive predictive value; NPV: negative predictive value; PLR: positive likelihood ratio; NLR: negative likelihood ratio; LB: lactate and bilirubin; SOFA: sequential organ failure assessment; CLIF-SOFA: chronic liver failure-sequential organ failure assessment; MELD: model for end-stage liver disease.

TABLE 5: Diagnostic accuracy of different scoring systems in predicting 3-year mortality at the optimal cutoff point.

\begin{tabular}{|c|c|c|c|c|c|c|c|c|c|}
\hline Prognostic models & AUROC $(95 \%$ CI) & $P$ value & Cutoff point & Sensitivity & Specificity & PPV & NPV & PLR & NLR \\
\hline MELD & $0.726(0.680-0.771)$ & - & 18 & 0.61 & 0.74 & 0.76 & 0.58 & 2.36 & 0.53 \\
\hline Child-Pugh & $0.602(0.552-0.652)$ & $<0.001$ & 10 & 0.76 & 0.41 & 0.64 & 0.55 & 1.28 & 0.59 \\
\hline SOFA & $0.683(0.635-0.730)$ & 0.045 & 10 & 0.53 & 0.73 & 0.73 & 0.53 & 1.97 & 0.64 \\
\hline CLIF-SOFA & $0.698(0.651-0.744)$ & 0.136 & 10 & 0.63 & 0.69 & 0.73 & 0.58 & 2.02 & 0.54 \\
\hline LB index & $0.694(0.648-0.741)$ & 0.159 & 6.18 & 0.51 & 0.82 & 0.79 & 0.55 & 2.88 & 0.59 \\
\hline
\end{tabular}

DeLong test was used to compare the AUC between MELD score and other clinical models. AUROC: area under the receiver operating characteristic curve; CI: confidence interval; PPV: positive predictive value; NPV: negative predictive value; PLR: positive likelihood ratio; NLR: negative likelihood ratio; MELD: model for end-stage liver disease; SOFA: sequential organ failure assessment; CLIF-SOFA: chronic liver failure-sequential organ failure assessment; LB: lactate and bilirubin.

has good prediction accuracy. The LB index can rapidly evaluate the severity of liver cirrhosis and show optimal discrimination ability to predict short-term prognosis in critically ill cirrhotic patients. In our research, we also compared the ability of several scoring systems including the LB index to predict both short- and long-term mortality in critically ill cirrhotic patients. The MELD score performed well in predicting both short- and long-term prognosis. Lactate can improve prediction of short-term mortality in critically ill patients with cirrhosis while its predictive value regarding long-term mortality requires further investigation [19]. The LB index was associated with lactate level, which may lead to the poor performance of LB index in predicting long-term mortality compared with its value in predicting short-term mortality. All need further study.

There are some potential clinically relevant applications of the new LB index. Cirrhotic patients with an LB index more than 6.18 have severely impaired liver function and a high risk of short-term death. An LB index more than 6.18 could be used as an indication for liver transplantation. It has been reported that the prognosis of cirrhotic patients with hepatocellular carcinoma (HCC) and the outcome of HCC treatments is linked to the appropriate assessment of liver function because some standard therapies could indirectly damage nontumoural liver parenchyma leading to hepatic decompensation [34, 35]. Only a few patients with 


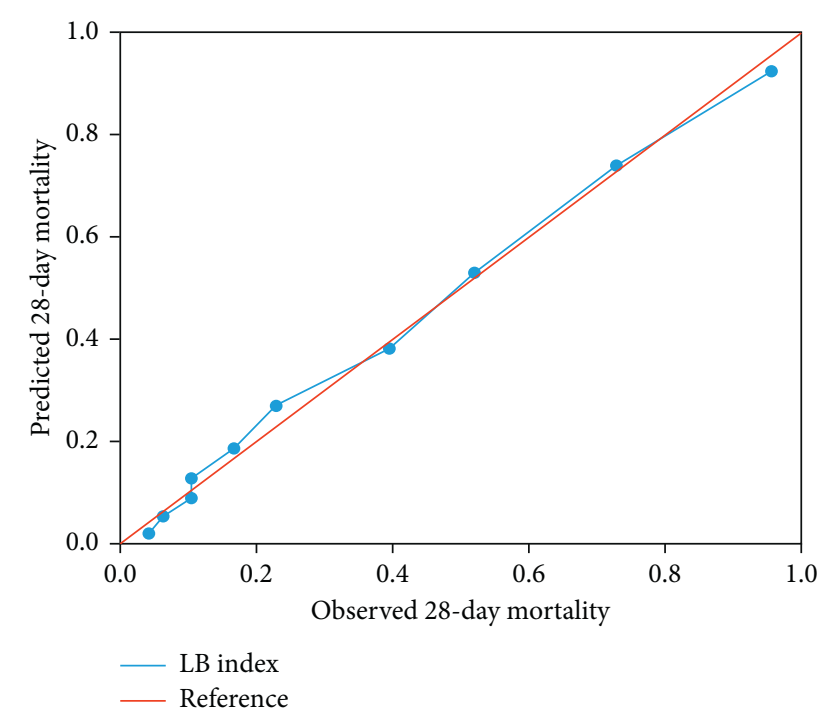

FIgURE 3: Calibration curve of the LB index for 28-day mortality. LB: lactate and bilirubin.

hepatocellular carcinoma in the Child-Pugh B class can benefit from liver transplantation and whether others will benefit from limited treatment option except liver transplantation remains uncertain [36], but a better definition of liver function by other prognostic scores such as ALBI grade can be useful to better select patients potentially suitable for HCC treatments [37-39]. The LB index could be a new adjunctive parameter to help patient selection. Further studies are needed to provide more useful information on this subject.

However, a few potential limitations of the research need our considerations. First, the research was a retrospective research, which was carried out in a single institution. Second, this study's mortality was defined as all-cause mortality, and other factors of death may affect critically ill cirrhotic patients' mortality. Finally, part of general ICU scoring systems and classic liver-specific scoring systems were included, but others were excluded. Problems will be addressed in our following studies.

\section{Conclusions}

The laboratory-based LB index performs well in predicting critically ill cirrhotic patients' short-term prognosis, while, for long-term prognosis, the MELD scoring system is more appropriate. In addition, the new LB index may have many potential clinically relevant applications, which need us to further explore and verify.

\section{Data Availability}

The data used to support the findings of this study are available from the corresponding author upon request.

\section{Conflicts of Interest}

The authors declare that there are no conflicts of interest regarding the publication of this paper.

\section{Authors' Contributions}

Xiao-Fu Chen, Yuan Zhao, Wei-Zhen Chen, and Zhi-Ming Huang put forward the research plan; Xiao-Fu Chen, Yuan Zhao, Wei-Zhen Chen, and Xin-Tian Shao were involved in data collection and analysis; Xiao-Fu Chen, Yuan Zhao, and Xin-Tian Shao wrote the manuscript; Xiao-Fu Chen, and Zhi-Ming Huang were involved in critical review and revision of the manuscript. All the authors made their own contributions and agreed to submit the manuscript.

\section{References}

[1] E. A. Tsochatzis, J. Bosch, and A. K. Burroughs, "Liver cirrhosis," The Lancet, vol. 383, no. 9930, pp. 1749-1761, 2014.

[2] A. J. O' Brien, C. A. Welch, M. Singer, and D. A. Harrison, "Prevalence and outcome of cirrhosis patients admitted to UK intensive care: a comparison against dialysis-dependent chronic renal failure patients," Intensive Care Med, vol. 38, pp. 9911000, 2012.

[3] O. Lerschmacher, A. Koch, K. Streetz, C. Trautwein, and F. Tacke, "Management der dekompensierten Leberzirrhose auf der Intensivstation," Medizinische Klinik-Intensivmedizin und Notfallmedizin, vol. 108, no. 8, pp. 646-656, 2013.

[4] F. Saliba, P. Ichaï, E. Levesque, and D. Samuel, "Cirrhotic patients in the ICU," Current Opinion in Critical Care, vol. 19, no. 2, pp. 154-160, 2013.

[5] E. Levesque, F. Saliba, P. Ichaï, and D. Samuel, "Outcome of patients with cirrhosis requiring mechanical ventilation in ICU," Journal of Hepatology, vol. 60, no. 3, pp. 570-578, 2014.

[6] I. Albers, H. Hartmann, J. Bircher, and W. Creutzfeldt, "Superiority of the Child-Pugh classification to quantitative liver function tests for assessing prognosis of liver cirrhosis," Scandinavian Journal of Gastroenterology, vol. 24, no. 3, pp. 269-276, 1989.

[7] J.-L. Vincent, R. Moreno, J. Takala et al., "The SOFA (Sepsisrelated Organ Failure Assessment) score to describe organ dysfunction/failure," Intensive Care Medicine, vol. 22, no. 7, p. 707, 1996.

[8] R. Moreau, R. Jalan, P. Gines et al., "Acute-on-chronic liver failure is a distinct syndrome that develops in patients with acute decompensation of cirrhosis," Gastroenterology, vol. 144, no. 7, pp. 1426-1437, Article ID e1429, 2013.

[9] P. Kamath, R. H. Wiesner, M. Malinchoc et al., "A model to predict survival in patients with end-stage liver disease," Hepatology, vol. 33, no. 2, pp. 464-470, 2001.

[10] D.-Q. Sun, C.-F. Zheng, W.-Y. Liu et al., "AKI-CLIF-SOFA: a novel prognostic score for critically ill cirrhotic patients with acute kidney injury," Aging, vol. 9, no. 1, p. 286, 2017.

[11] H. C. Pan, C. C. Jenq, M. H. Tsai et al., "Scoring systems for 6month mortality in critically ill cirrhotic patients: a prospective analysis of chronic liver failure sequential organ failure assessment score (CLIF-SOFA)," Alimentary Pharmacology \& Therapeutics, vol. 40, pp. 1056-1065, 2015.

[12] H. C. Pan, C. C. Jenq, M. H. Tsai et al., "Short-term and longterm vital outcomes of cirrhotic patients admitted to an intensive care unit," The European Journal of Gastroenterology \& Hepatology, vol. 22, pp. 1474-1480, 2010.

[13] J. A. Kraut and N. E. Madias, "Lactic acidosis," New England Journal of Medicine, vol. 371, no. 24, pp. 2309-2319, 2014.

[14] J. B. Jeppesen, C. Mortensen, F. Bendtsen, and S. Møller, "Lactate metabolism in chronic liver disease," Scandinavian 
Journal of Clinical and Laboratory Investigation, vol. 73, no. 4, pp. 293-299, 2013.

[15] B. Scheiner, G. Lindner, T. Reiberger et al., "Acid-base disorders in liver disease," Journal of Hepatology, vol. 67, no. 5, pp. 1062-1073, 2017

[16] N. D. Murphy, S. K. Kodakat, J. A. Wendon et al., "Liver and intestinal lactate metabolism in patients with acute hepatic failure undergoing liver transplantation," Critical Care Medicine, vol. 29, no. 11, pp. 2111-2118, 2001.

[17] J. A. Kruse, S. A. J. Zaidi, and R. W. Carlson, "Significance of blood lactate levels in critically III patients with liver disease," The American Journal of Medicine, vol. 83, no. 1, pp. 77-82, 1987.

[18] F. S. Cardoso, J. G. Abraldes, E. Sy et al., "Lactate and number of organ failures predict intensive care unit mortality in patients with acute-on-chronic liver failure," Liver International, vol. 39, 2019.

[19] A. Drolz, T. Horvatits, K. Rutter et al., "Lactate improves prediction of short-term mortality in critically ill patients with cirrhosis: a multinational study," Hepatology, vol. 69, no. 1, pp. 258-269, 2019.

[20] C. Edmark, M. J. W. McPhail, M. Bell, T. Whitehouse, J. Wendon, and K. B. Christopher, "LiFe: a liver injury score to predict outcome in critically ill patients," Intensive Care Medicine, vol. 42, no. 3, pp. 361-369, 2016.

[21] A. E. W. Johnson, T. J. Pollard, L. Shen et al., "MIMIC-III, a freely accessible critical care database," Science Data, vol. 3, Article ID 160035, 2016.

[22] A. Drolz, B. Jäger, M. Wewalka et al., "Clinical impact of arterial ammonia levels in ICU patients with different liver diseases," Intensive Care Medicine, vol. 39, no. 7, pp. 1227-1237, 2013.

[23] K. Staufer, K. Roedl, D. Kivaranovic et al., "Renal replacement therapy in critically ill liver cirrhotic patients-outcome and clinical implications," Liver International, vol. 37, no. 6, pp. 843-850, 2017.

[24] E. R. Delong, D. M. Delong, and D. L. Clarke-Pearson, "Comparing the areas under two or more correlated receiver operating characteristic curves: a nonparametric approach," Biometrics, vol. 44, no. 3, pp. 837-845, 1988.

[25] K. Roedl, C. Wallmüller, A. Drolz et al., "Outcome of in- and out-of-hospital cardiac arrest survivors with liver cirrhosis," The Annals of Intensive Care, vol. 7, p. 103, 2017.

[26] A. Drolz, T. Horvatits, K. Roedl et al., "Coagulation parameters and major bleeding in critically ill patients with cirrhosis," Hepatology, vol. 64, no. 2, pp. 556-568, 2016.

[27] F. L. Ferreira, D. P. Bota, A. Bross, C. Mélot, and J. L. Vincent, "Serial evaluation of the SOFA score to predict outcome in critically ill patients," JAMA, vol. 286, no. 14, pp. 1754-1758, 2001.

[28] J. Bakker, M. W. Nijsten, and T. C. Jansen, "Clinical use of lactate monitoring in critically ill patients," Annals of Intensive Care, vol. 3, no. 1, p. 12, 2013.

[29] S. A. Haas, T. Lange, B. Saugel et al., "Severe hyperlactatemia, lactate clearance and mortality in unselected critically ill patients," Intensive Care Medicine, vol. 42, no. 2, pp. 202-210, 2016.

[30] A. D. Nichol, M. Egi, V. Pettila et al., "Relative hyperlactatemia and hospital mortality in critically ill patients: a retrospective multi-centre study," Critical Care, vol. 14, no. 1, p. R25, 2010.

[31] P. L. Almenoff, J. Leavy, M. H. Weil, N. B. Goldberg, D. Vega, and E. C. Rackow, "Prolongation of the half-life of lactate after maximal exercise in patients with hepatic dysfunction," Critical Care Medicine, vol. 17, no. 9, pp. 870-873, 1989.

[32] G. C. Macquillan, M. S. Seyam, P. Nightingale, J. M. Neuberger, and N. Murphy, "Blood lactate but not serum phosphate levels can predict patient outcome in fulminant hepatic failure," Liver Transplantation, vol. 11, no. 9, pp. 1073-1079, 2005.

[33] G. C. Funk, D. Doberer, N. Kneidinger, G. Lindner, U. Holzinger, and B. Schneeweiss, "Acid?base disturbances in critically ill patients with cirrhosis," Liver International, vol. 27, no. 7, pp. 901-909, 2007.

[34] J. Bruix and M. Sherman, "Management of hepatocellular carcinoma: an update," Hepatology, vol. 53, no. 3, pp. 1020-1022, 2011.

[35] J. Bruix, M. Reig, and M. Sherman, "Evidence-based diagnosis, staging, and treatment of patients with hepatocellular carcinoma," Gastroenterology, vol. 150, no. 4, pp. 835-853, 2016.

[36] A. Granito and L. Bolondi, "Non-transplant therapies for patients with hepatocellular carcinoma and Child-PughTurcotte class B cirrhosis," The Lancet Oncology, vol. 18, no. 2, pp. e101-e112, 2017.

[37] P. J. Johnson, S. Berhane, C. Kagebayashi et al., “Assessment of liver function in patients with hepatocellular carcinoma: a new evidence-based approach-the ALBI grade," Journal of Clinical Oncology, vol. 33, no. 6, pp. 550-558, 2015.

[38] D. J. Pinato, R. Sharma, E. Allara et al., "The ALBI grade provides objective hepatic reserve estimation across each BCLC stage of hepatocellular carcinoma," Journal of Hepatology, vol. 66, no. 2, pp. 338-346, 2017.

[39] M. Deng, S. W. Y. Ng, S. T. Cheung, and C. C. N. Chong, "Clinical application of albumin-bilirubin (ALBI) score: the current status," The Surgeon, vol. 18, no. 3, pp. 178-186, 2020. 